Ультразвук легких у пациентов

с пневмонией, вызванной

COVID-19: сравнение с данными

компьютерной томографии. Обсервационное проспективное

\section{клиническое исследование}

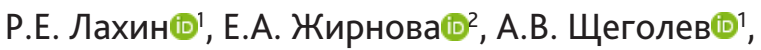 \\ О. Йованикич(D) ${ }^{3}$ И.С. Железняк(1) И.А. Меньков (D1, \\ В.В. Салухов ${ }^{1}$, А.А. Чугунов ${ }^{1}$ \\ 1 ФГБВОУВО «Военно-медицинская академия имени \\ С.М. Кирова» МО РФ, Санкт-Петербург, Россия \\ 2 Клиника высоких медицинских технологий им. Н.И. Пирогова \\ Санкт-Петербургского государственного университета, \\ Санкт-Петербург, Россия \\ ${ }^{3}$ Военно-медицинская академия, Белград, Сербия
}

\section{Peфераm}

Актуальность. Наиболее тяжелой формой новой коронавирусной инфекции COVID-19 является пневмония. Большая площадь повреждения, высокая летальность при COVID-19 ставят задачу быстрой прикроватной диагностики и динамического мониторинга объема и характера повреждения легочной ткани. Таким инструментом стало ультразвуковое исследование (УЗИ) легких.

Цель исследования. Сравнение данных, получаемых при компьютерной томографии (КТ) и УЗИ легких, а также точности ультразвуковой посегментной верификации зон повреждения у пациентов с пневмонией, вызванной коронавирусом тяжелого острого респираторного синдрома - 2 (SARS-CoV-2).

Материалы и методы. В обсервационное проспективное клиническое исследование включено 388 пациентов в возрасте 18-75 лет с подтвержденным диагнозом пневмонии, вызванной COVID-19, или подозрением на COVID-19. УзИ легких выполняли в течение 24 ч после проведения КТ органов грудной клетки. При проведении КТ определяли патологические признаки инфильтрации и консолидации легких, которые регистрировали по сегментам легких. УЗИ легких проводили по «Русскому протоколу», ультразвуковые признаки В-линий и консолидации также регистрировали на основании проекции

\section{Lung ultrasound in COVID-19 pneumonia: comparison with computed tomography. An observation prospective clinical trial}

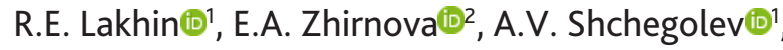 \\ O. Jovanikić(iD ${ }^{3}$, I.S. Zheleznyak (D), I.A. Menkovid, \\ V.V. Salukhovid', A.A. Chugunovi(1) \\ ${ }^{1}$ Military Medical Academy, St. Petersburg, Russia \\ 2 Saint Petersburg State University, St. Petersburg, Russia \\ ${ }^{3}$ Military Medical Academy, Belgrade, Serbia
}

\section{Abstract}

Introduction. Pneumonia is the most severe form of the new coronavirus infection (COVID-19). The large area of damage and the high mortality rate in COVID-19 pose the challenge of rapid bedside diagnosis and dynamic monitoring of the volume and nature of lung tissue damage.

Objectives. The goal of the study was to compare the data obtained with computed tomography and ultrasound of the lungs, as well as the accuracy of ultrasound segmentby-segment verification of damage zones in patients with pneumonia caused by SARS-CoV-2.

Material and methods. The observational prospective clinical study included 388 patients aged 18-75 years; with a confirmed diagnosis of pneumonia caused by COVID-19 or suspected COVID-19. Lung ultrasound was performed within 24 hours after computed tomography (CT) of the chest. During the CT scan, pathological signs, infiltration, and consolidation of the lungs were determined, which were recorded by lung segments. Ultrasound of the lungs was performed according to the "Russian Protocol", ultrasound signs in the lines and consolidation were also recorded based on the projection of the lung segments on the chest wall. An analysis was performed to describe and generalize the distributions of variables. The sensitivity and specificity of ultrasound methods were evaluated with ROC analysis against CT as a "gold standard".

Results. Bilateral involvement was found in $100 \%$ of cases. Typical signs of pneumonia caused by coronavirus infection on CT were a "ground glass" pattern, thickened 
сегментов легких на грудную стенку. Был проведен анализ для описания и обобщения распределений переменных. Оценку чувствительности и специфичности ультразвуковых методов проводили на основании ROC-анализа по «золотому стандарту» КТ.

Результаты. Двустороннее вовлечение было обнаружено в 100 \% случаев. Характерными для пневмонии, вызванной коронавирусной инфекцией, признаками на КТ стали уплотнение легочной паренхимы по типу «матового стекла», утолщенная плевра, консолидация, ретикулярные уплотнения интерстиция, симптом «булыжной мостовой». При УзИ легких и плевры выявляемые признаки соответствовали признакам КТ. Наиболее часто при УЗИ встречались В-линии (мультифокальные, дискретные или сливающиеся) и консолидация различных объемов легочной ткани. Чувствительность УЗИ легких в точности посегментной диагностики повреждения признаком инфильтрации составила 87,9 \%, а специфичность - 91,5 \% (площадь под ROC-кривой 0,939, $p<0,001$ ). Чувствительность ультразвукового признака консолидации составила 84,6 \%, специфичность - 79,2 \% (площадь под ROC-кривой 0,846, $p<0,001)$.

Заключение. Использование УЗИ легких у пациентов с COVID-19 позволяет выявить оценить объем и характер повреждения легочной ткани. Таким образом, УзИ легких продемонстрировало точность, сравнимую с КТ органов грудной клетки, при выявлении повреждения легких у пациентов с COVID-19.

Ключевые слова: коронавирусная инфекция, COVID-19, УЗИ легких, компьютерная томография, УзИ, пневмония

\ Для корреспонденции: Лахин Роман Евгеньевич д-р мед. наук, профессор кафедры анестезиологии и реаниматологии ФГБВОУ ВО «Военно-медицинская академия им. С.М. Кирова» МО РФ, Санкт-Петербург, Россия; e-mail: doctor-lahin@yandex.ru

匹 Для цитирования: Лахин Р.Е., Жирнова Е.А., Щеголев А.В., Йованикич О., Железняк И.С., Меньков И.А., Салухов В.В., Чугунов А.А. Ультразвук легких у пациентов с пневмонией, вызванной COVID-19: сравнение с данными компьютерной томографии. Обсервационное проспективное клиническое исследование. Вестник интенсивной терапии им. А.И. Салтанова. 2021;2:82-93. DOI: 10.21320/1818-474X-2021-2-82-93

ए Поступила: 15.03.2021

ㅁ. Принята кпечати: 02.06.2021 pleura, consolidation, reticular pattern, and a "crazy paving" pattern. During ultrasound examination of the lungs and pleura, the detected signs corresponded to the signs of CT. $\mathrm{B}$-lines (multifocal, discrete, or confluent) and consolidation of various volumes of lung tissue were most frequently encountered during ultrasound. The sensitivity of ultrasound of the lungs in the accuracy of the segment-by-segment diagnosis of damage by the sign of infiltration is $87.9 \%$, and the specificity is $91.5 \%$ (the area under the ROC curve is $0.939 ; p<0.001)$. The sensitivity of the ultrasonic sign of consolidation was $84.6 \%$, and the specificity was $79.2 \%$ (the area under the ROC curve is 0.846; $p<0.001$ ).

Conclusions. The use of ultrasound of the lungs during the COVID-19 allows us to identify and assess the volume and nature of lung damage. Thus, lung ultrasonography has demonstrated accuracy comparable to chest $\mathrm{CT}$ in detecting lung damage in patients with COVID-19.

Keywords: coronavirus disease, COVID-19, lung ultrasonography, computed tomography, ultrasound, pneumonia

$\triangle$ For correspondence: Roman E. Lakhin - Doctor of Medical Science, Professor, Department of Anesthesiology and Intensive Care, Military Medical Academy, St. Petersburg, Russia; e-mail: doctorlahin@yandex.ru

[4 For citation: Lakhin R.E., Zhirnova E.A., Shchegolev A.V., Jovanikić O., Zheleznyak I.S., Menkov I.A., Salukhov V.V., Chugunov A.A. Lung ultrasound in COVID-19 pneumonia: comparison with computed tomography. An observation prospective clinical trial. Annals of Critical Care. 2021;2:82-93. DOI: 10.21320/1818-474X-2021-2-82-93

匹 Received: 15.03 .2021

自 Accepted: 02.06.2021 


\section{Введение}

Интерес к ультразвуковому исследованию (УЗИ) легких растет год от года, особенно в периоды вспышек эпидемий вирусной инфекции. Так было и при «свином», «птичьем» гриппе, и в настоящее время при новой коронавирусной инфекции [1-3].

Появление новой угрозы поставило перед специалистами задачи, в первую очередь связанные с быстрой диагностикой инфекции и заболевания. Наиболее тяжелой клинической формой стала пневмония, которая при коронавирусной инфекции COVID-19 чаще всего становилась причиной летальных исходов [4]. В ряде исследований было показано, что ранняя диагностика пневмонии с помощью компьютерной томографии (КТ), УЗИ еще до получения результатов полимеразной цепной реакции (ПЦР) может ускорить назначение лечения и способствовать своевременной изоляции пациента $[5,6]$. Роль УЗИ легких возрастает, если проведение пациентам радиологических методов исследования (КТ, рентгенография) нежелательно - например, у беременных женщин [7]. В этом случае УЗИ легких может стать приоритетным инструментом, с помощью которого будет получена информация об объеме и характере поражения легочной ткани [8].

Особенностью ультразвука является способность определять изменения, влияющие на соотношение между тканью и воздухом в легочной ткани. В норме под плеврой находятся альвеолы, заполненные воздухом, поэтому ультразвуковые (УЗ) волны полностью отражаются, создавая специфический реверберационный артефакт горизонтальных линий, который получил название А-линии. Когда соотношение между воздухом, тканью, жидкостью или другими биологическими компонентами уменьшается, то легкое перестает практически полностью отражать УЗ-волну, и проникающий между воздушными альвеолами ультразвук приводит к появлению вертикальных артефактов, которые были названы В-линиями. Субплевральная ткань с плотностью, близкой к значению 1 г/мл, выглядит как область, в которой воздушная среда отсутствует и ультразвук проникает в эти участки беспрепятственно, формируя артефакт, получивший название консолидации, или тканевого признака [9-13].

При прогрессировании пневмонии при COVID-19 изменения в паренхиме легких начинаются в дистальных отделах легкого и прогрессируют проксимально. На ранних стадиях заболевания наблюдаются изменения в виде помутнения - «матового стекла» и «булыжной мостовой», наблюдаемые при КТ, а позже - более крупные уплотнения в базальных или зависимых отделах легких $[5,14,15]$. Таким образом, патофизиологические изменения в легких, развивающиеся при пневмонии COVID-19, хорошо подходят для такого метода визуализации, как УЗИ легких. Однако подходы к УЗ-визуализации у разных исследователей различаются, как различаются и методики оценки обнаруженных изменений $[5,6,11,16,17]$.

Целью исследования стало сравнение данных, получаемых при КТ и УЗИ легких у пациентов с пневмонией, вызванной COVID-19.

\section{Материалы и методы}

Обсервационное проспективное клиническое исследование проводили в ФГБВОУ ВО «Военно-медицинская академия имени С.М. Кирова» МО РФ. Исследование было одобрено независимым этическим комитетом (протокол № 236 от 21.05.2020).

Критериями включения в исследование были возраст пациентов 18-75 лет, развитие внебольничной пневмонии, период между проведением КТ и УЗИ легких не более 24 ч; подтверждение COVID-19 или подозрение на COVID-19. Критерии невключения: наличие пневмоторакса, травма грудной клетки или операция на легких в анамнезе, фоновые специфические заболевания (туберкулез, саркоидоз).

Всего в исследовании приняло участие 388 пациентов. Общая характеристика пациентов при поступлении представлена в табл. 1.

При проведении КТ органов грудной полости определяли патологические признаки инфильтрации и консолидации легких, которые регистрировали по сегментам легких. Для верификации бронхолегочных сегментов на КТ использовали нумерацию и анатомическую номенклатуру, принятую Лондонским международным конгрессом отоларингологов в 1949 г. и Международным Парижским конгрессом анатомов в 1955 г. На срезах КТ сегменты легких определяли на основе схемы бронхиального дерева, границы сегментов находили по ветвям легочных вен (рис. 1). В правом легком определяли 10 сегментов; в левом легком $1+2$ сегмент представляет собой комбинацию за счет наличия общего бронха; также объединили 7-й и 8-й сегменты (поскольку отдельный бронх 7-го сегмента встречается не более чем в 30 \% случаев). Объединенные сегменты считали как один. Таким образом, в левом легком насчитывали 8 сегментов, и общее число сегментов составляло 18.

Прикроватное УЗИ проводили с помощью портативного У3-аппарата Mindray M7 (Китай), конвексным датчиком C5-2s (2,5-5 МГц). Процедура получения изображения была стандартизирована с использованием предустановки абдоминального исследования, максимальная глубина $18 \mathrm{~cm}$, фокусировка на плевральной линии. Усиление регулировалось для получения наилучшего изображения плевры, вертикальных артефактов и субплевральных консолидаций с воздушными бронхограммами или без них. Все гармоники и программное обеспечение для снижения артефактов были отключе- 
Таблица 1. Характеристика пациентов, включенных в исследование

Table 1. Characteristics of the study patients

\begin{tabular}{|c|c|c|}
\hline \multicolumn{2}{|r|}{ Показатель } & Значение \\
\hline \multicolumn{2}{|l|}{ Возраст, лет } & $52,6 \pm 10,5$ \\
\hline \multicolumn{2}{|l|}{ Пол (мужчины/женщины), \% } & 267/121 \\
\hline \multicolumn{2}{|l|}{ Рост, см } & $173,4 \pm 12,4$ \\
\hline \multicolumn{2}{|l|}{ Вес, кг } & $94,2 \pm 17$ \\
\hline \multicolumn{2}{|l|}{ Индекс массы тела, кг/м² } & $31,2 \pm 4,7$ \\
\hline \multicolumn{2}{|l|}{ День болезни при поступлении, $n$} & $5,4 \pm 2,0$ \\
\hline \multicolumn{2}{|c|}{ Среднетяжелая/тяжелая форма пневмонии, $n$} & $184 / 204$ \\
\hline \multicolumn{2}{|c|}{ Доля пациентов с исходно сниженной (< 94 \%) сатурацией при дыхании атмосферным воздухом, $n$ (\%) } & $252(64,9 \%)$ \\
\hline \multirow[t]{3}{*}{ Степень повреждения при КТ } & 2 & $207(53,4)$ \\
\hline & 3 & $156(40,2)$ \\
\hline & 4 & $25(6,4)$ \\
\hline \multirow[t]{5}{*}{ Сопутствующие заболевания } & Сахарный диабет, $n$ (\%) & $78(20,1)$ \\
\hline & Ожирение, $n$ (\%) & $193(49,7)$ \\
\hline & Гипертоническая болезнь, $n$ (\%) & $252(64,9)$ \\
\hline & Ишемическая болезнь сердца, $n$ (\%) & $95(24,5)$ \\
\hline & Хроническая сердечная недостаточность, $n$ (\%) & $20(24,5)$ \\
\hline
\end{tabular}

ны. Грудную клетку исследовали в положении лежа или полусидя, в зависимости от состояния пациента. При проведении УЗИ определяли патологические признаки; признаки инфильтрации и консолидации легких регистрировали по сегментам легких.

УЗИ легких проводили в течение 24 ч после выполнения КТ согласно «Русскому протоколу» в 8 зонах справа и слева - итого 16 зон (рационализаторское предложение № 15165, протокол ВМедА № 4 от 15.12.2020) (рис. 2) [18].

Предложенный протокол имеет анатомический принцип формирования зон сканирования. Проекция сегментов легких на зоны «Русского протокола» представлена на рис. 3. При УЗИ слева при выявлении изменений в зонах проекции 1-го и 2-го сегмента регистрировали их как 1 сегмент (соответственно схемы КТ 1+2).

\section{Статистическая обработка}

Анализ данных проводился с помощью программы SPSS-26 для Windows (Statistical Package for Social Science, SPSS Inc. Chicago, IL, USA). Переменные были исследованы с помощью визуальных (гистограммы, вероятностный график) и аналитических методов (критерий Колмогорова-Смирнова / Шапиро-Уилка) для опре- деления нормальности распределения. При нормальном распределении данные были представлены средним значением и стандартным отклонением M (SD); количественные данные, не подчиняющиеся закону нормального распределения, представляли в виде медианы и 25-75-го перцентилей - Me (Q1-Q3). Был проведен анализ для описания и обобщения распределений переменных. Описание частот значения в исследуемой выборке представлено с обязательным указанием на приводимую характеристику выборки ( $n, \%)$. Межгрупповые сравнения в отношении номинальных данных проводили с использованием U-критерия Манна-Уитни. Оценку чувствительности (Se), специфичности (Sp) У3-методов проводили на основании ROC-анализа по «золотому стандарту» КТ. Выполняли визуальную оценку ROC-кривых, рассчитывали показатели чувствительности и специфичности, а также определяли площадь под ROC-кривой и ее координатные точки. Статистически значимыми считали тесты при $p<0,05$.

\section{Результаты исследования}

В исследование были включены 388 пациентов (267 мужчин, 121 женщина; средний возраст $52,6 \pm 10,5$ лет). Наиболее частыми симптомами при 

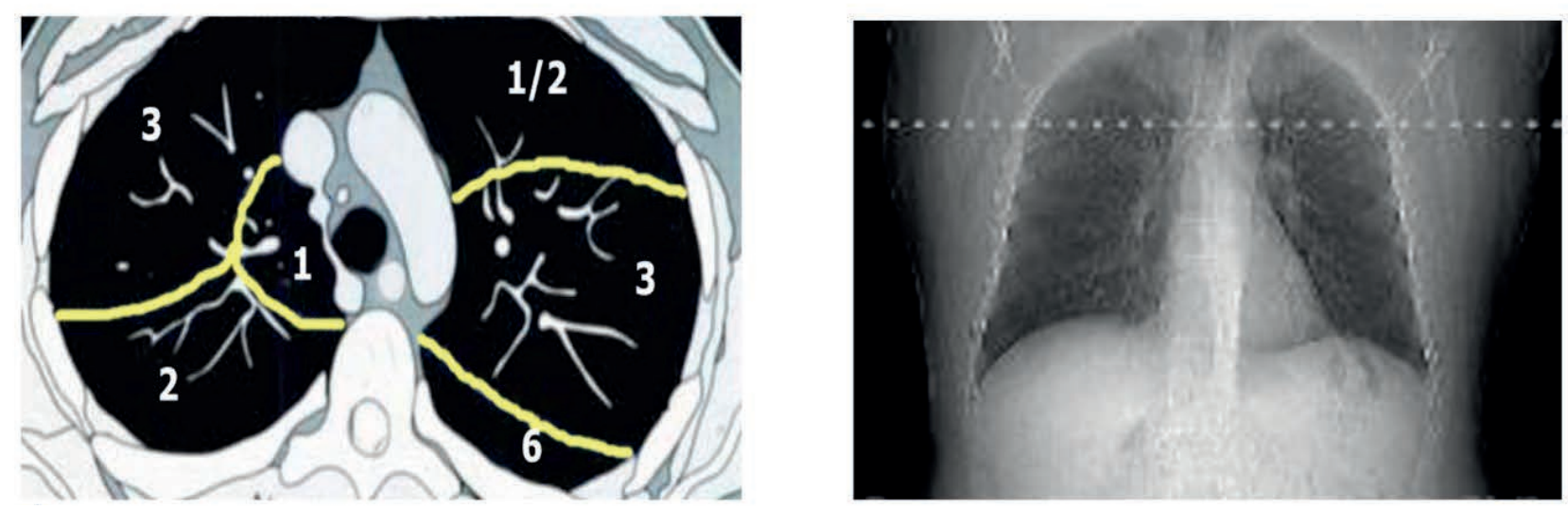

A
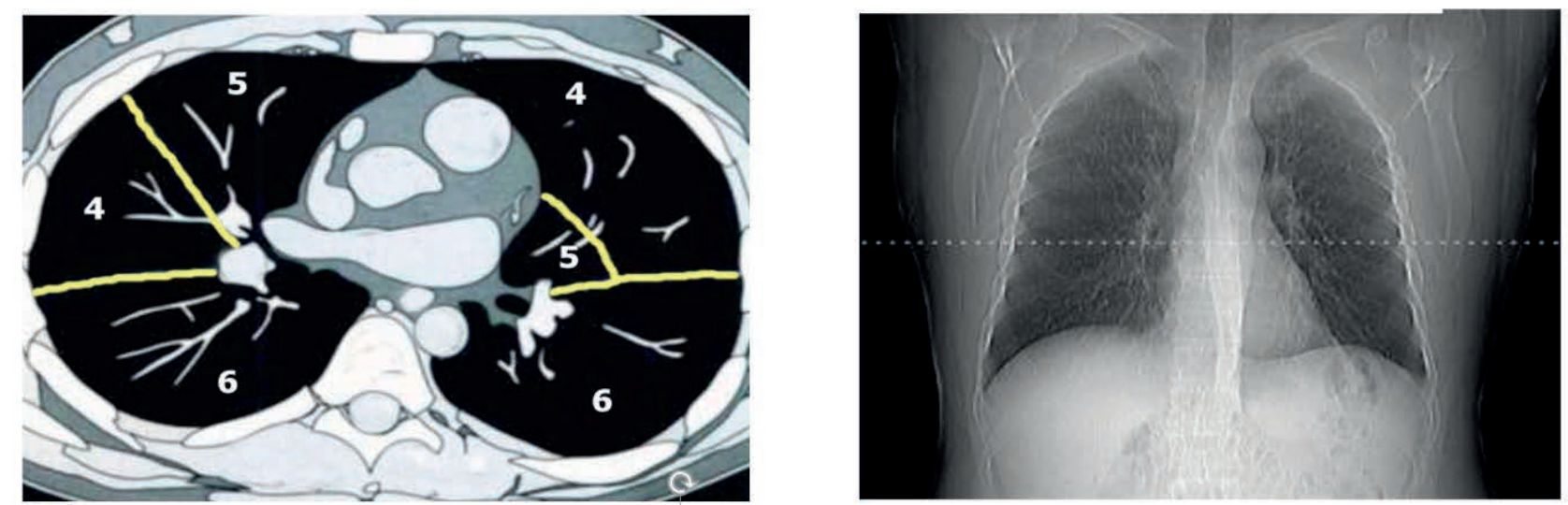

Б
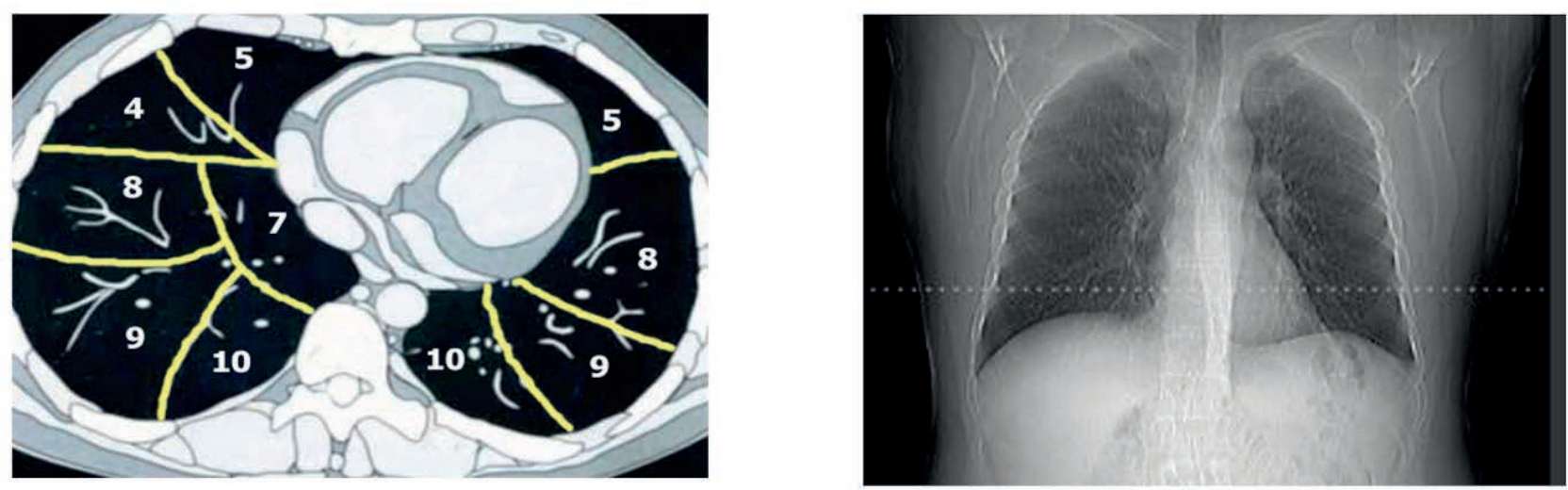

B

Рис. 1. Срезы сканирования КТ органов грудной полости: $A-$ на уровне верхней доли; $5-$ на уровне средней доли; $B-$ на уровне нижней доли

Fig. 1. Cross-sectional tomographic images of the body. $A$ - tomographic images of upper lobe; $\overline{-}$ - tomographic images of middle lobe; $B$ - tomographic images of lower lobe 


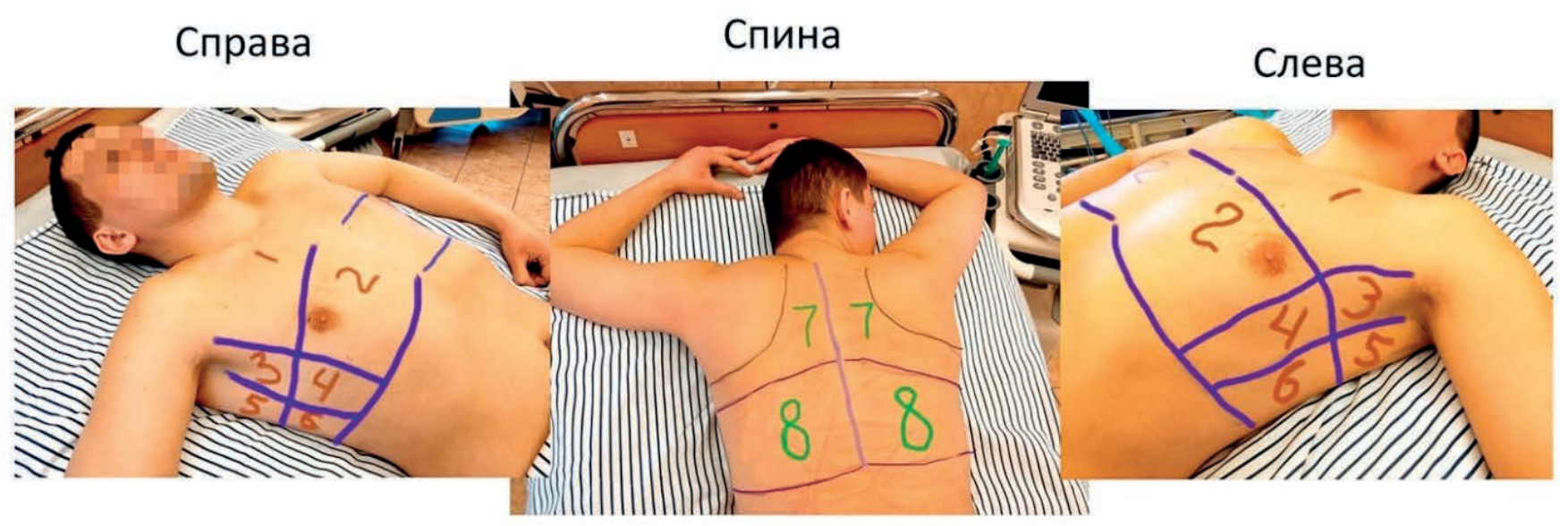

Рис. 2. Схема зон «Русского протокола» ультразвукового осмотра в положении лежа

Fig. 2. Zones of "Russian protocol". Ultrasound examination in the supine and prone position
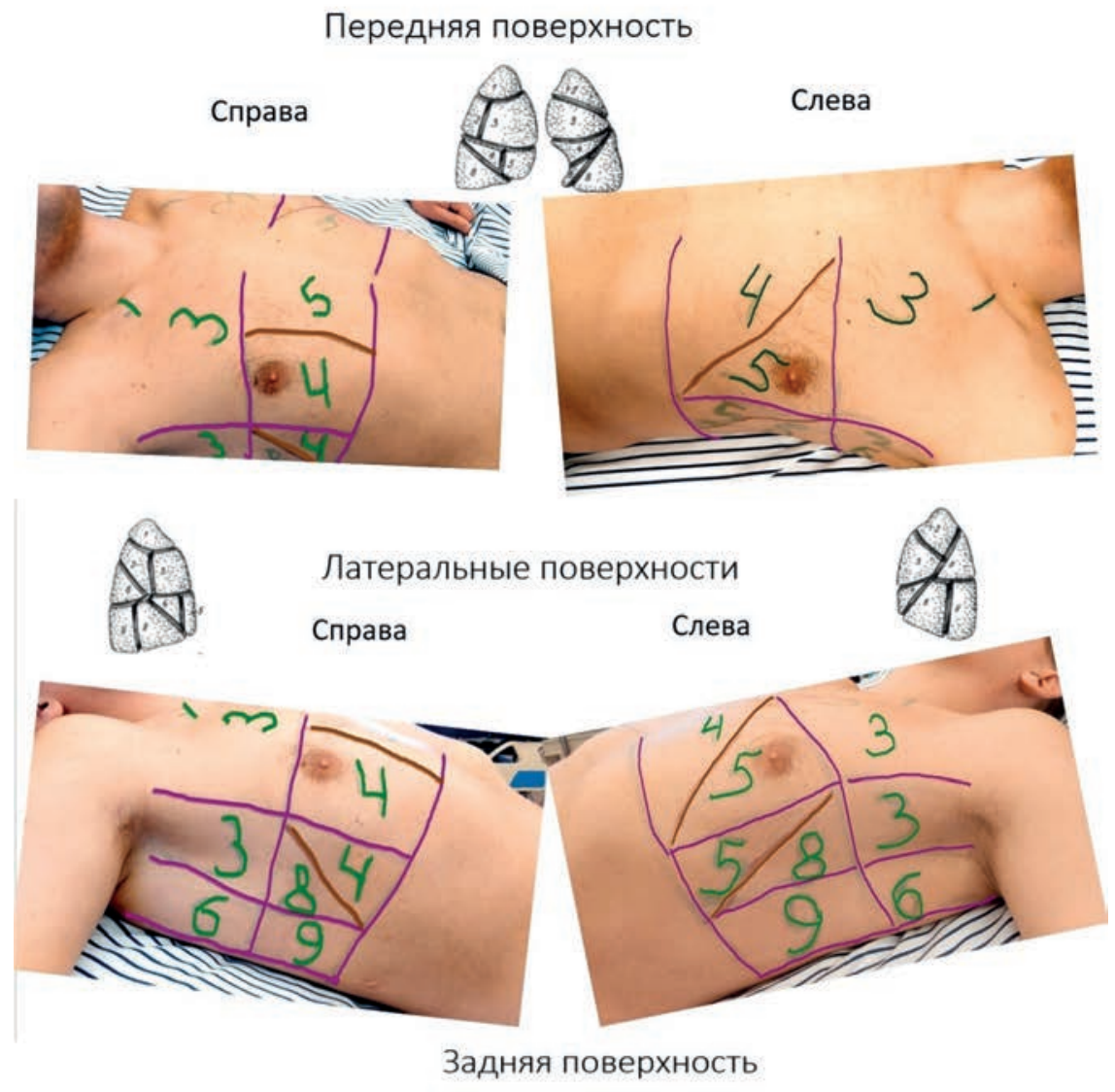

Рис. 3. Проекция сегментов легких на зоны «Русского протокола»

Fig. 3. Lung's segments on the zones of "Russian protocol"

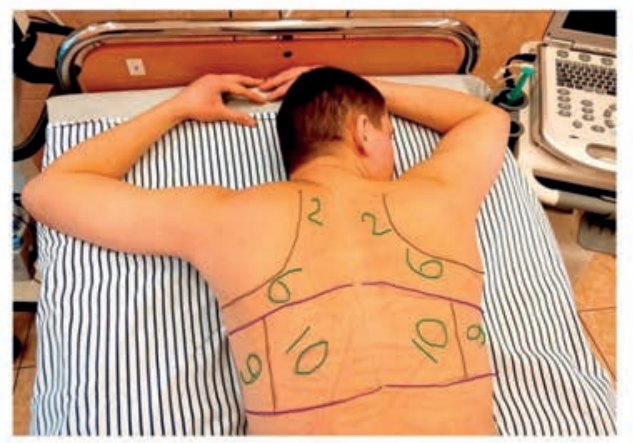


поступлении были лихорадка (372; 95,9 \%), кашель (114; $80,9 \%)$ и одышка $(147 ; 37,9$ \%). Длительность симптомов перед поступлением в стационар составила в среднем 5,4 $\pm 2,0$ дня. В кислородной поддержке нуждались 252 (64,9 \%) пациента. Исходные клинические характеристики пациентов представлены в табл. 1. Наиболее частыми сопутствующими заболеваниями стали гипертоническая болезнь (252; 64,9\%), ожирение (193; 49,7 \%), сахарный диабет (78; 20,1 \%), ишемическая болезнь сердца и хроническая сердечная недостаточность $(95 ; 24,5 \%)$.

В исследование были включены пациенты, у которых при проведении КТ подтвердилось развитие вирусной или вирусно-бактериальной пневмонии. Пациентов с единичными очагами инфильтрации легких в исследование не включали. По степени повреждения легких по данным КТ пациенты распределились: КТ-2 207 (53,4 \%); КТ-3 - 156 (40,2 \%); КТ-4 - 25 (6,4 \%) (см. табл. 1). Двустороннее вовлечение было обнаружено в $100 \%$ случаев.

Характерными для пневмонии, вызванной коронавирусной инфекцией, признаками на КТ стали уплотнение легочной паренхимы по типу «матового стекла», утолщенная плевра, консолидация, ретикулярные уплотнения интерстиция, симптом «булыжной мостовой». При УЗИ легких и плевры выявляемые признаки соответствовали признакам КТ. Если при КТ обнару- живали утолщение плевры, то при УЗИ также визуализировали утолщенную плевральную линию. Наиболее часто при КТ встречалось уплотнение легочной паренхимы по типу «матового стекла», которому при УЗИ соответствовали В-линии (мультифокальные, дискретные или сливающиеся). Если ретикулярные уплотнения интерстиция достигали плевры или зоны консолидации, то при УЗИ они определялись как В-линии (чаще дискретные).

Консолидацию легочной ткани при КТ определяли как в субплевральной зоне, так и в виде центральных зон консолидации, а также небольших центральных зон консолидации внутри участка уплотнения легочной паренхимы по типу «матового стекла». Для визуализации в ходе УЗИ легких и плевры доступны те зоны консолидации, которые прилегают к плевре, а центрально расположенная консолидация будет закрыта УЗ-артефактом воздушного (А-линии) или инфильтрированного (В-линии) легкого. Так, на рис. 4 на срезе КТ показана периферическая инфильтрация с центральным расположением воздушной ткани и зоной консолидации внутри инфильтрированной ткани, а при УЗИ будут определяться В-линии во всех исследуемых зонах. На рис. 4, $Б$, периферическая воздушная ткань с центральным расположением инфильтрации и участка консолидированной ткани при УЗИ будет визуализироваться как признак А-линий либо В-линий.
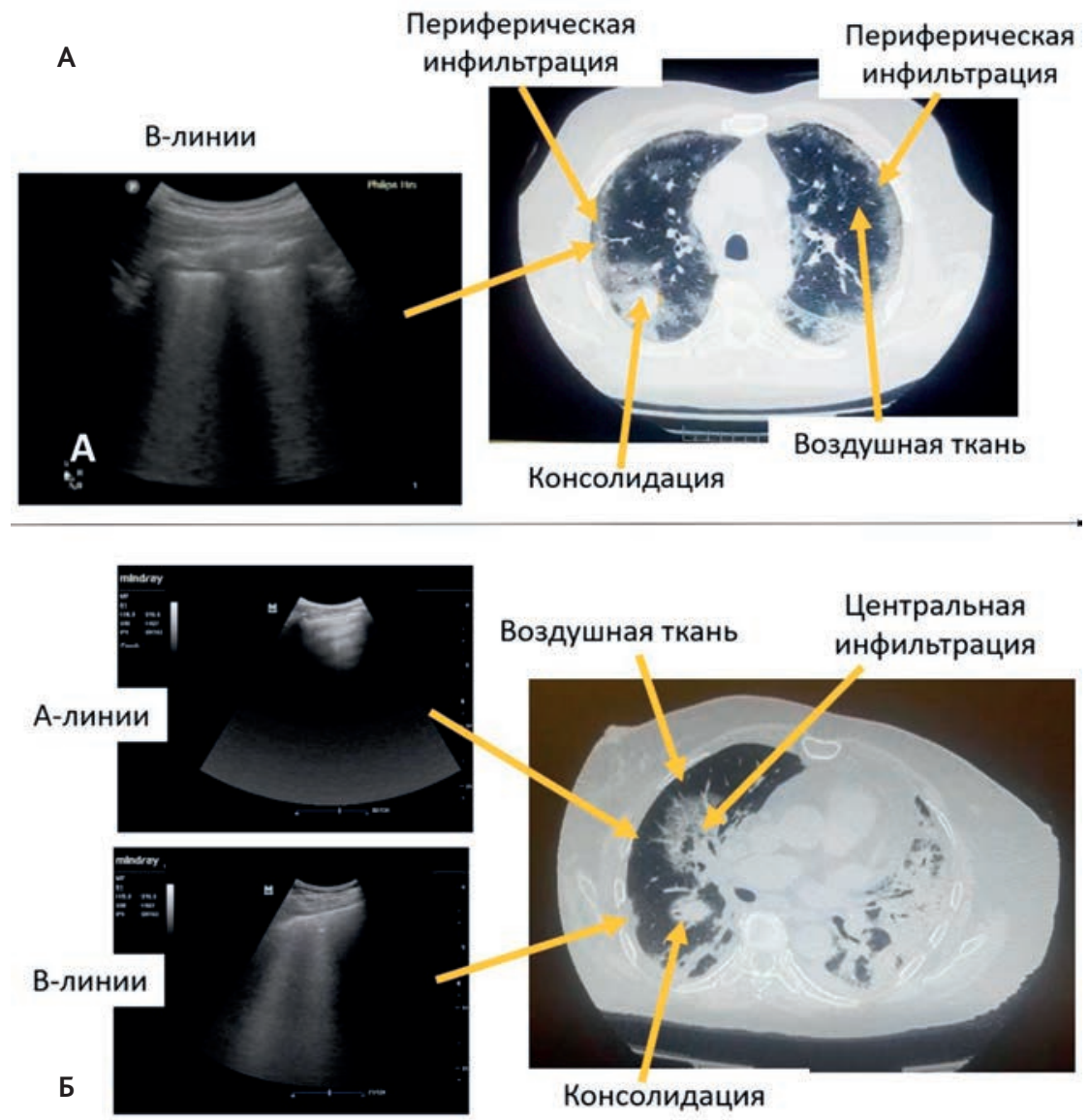

Рис. 4. Проблемы, возникающие при ультразвуковой визуализации консолидаций. $A-$ периферическая инфильтрация с центральным расположением воздушной ткани; Бпериферическая воздушная ткань с центральным расположением инфильтрации

Fig. 4. Problems with ultrasound imaging of consolidation. $A$ - peripheral lesion with aerated tissue in the center; 5 - peripheral air tissue with lesion in the center 
Если при КТ на фоне признака консолидации определялись воздушные бронхи, то при УЗИ внутри зон консолидации визуализировали признак воздушной бронхограммы, который, как правило, носил динамический характер. Симптом «булыжной мостовой» при КТ обусловлен сочетанием признака «матового стекла» с утолщенными междольковыми перегородками, это комбинация при УЗИ легких характеризовалась комбинацией В-линий, перемежаю- щихся с небольшой субплевральной консолидацией в местах утолщения перегородок и поражения альвеол

Свободная жидкость в плевральной полости выявлялась редко и была связана с тяжелым, длительным заболеванием, присоединением бактериальной микрофлоры и декомпенсированной сердечной недостаточностью. Соответствие признаков, выявляемых при КТ и УЗИ плевры и легких представлены в табл. 2.

Таблица 2. Сопоставление признаков, выявляемых при КТ и УзИ легких и плевры

Table 2. Comparison CT signs and ultrasound findings in the lungs

\begin{tabular}{|ll|}
\hline \multicolumn{1}{|c|}{ кт легких } & \multicolumn{1}{c|}{ Узи легких } \\
\hline Утолщенная плевра & Утолщенная плевральная линия \\
\hline Уплотнение легочной паренхимы по типу «матового стекла» & В-линии (мультифокальные, дискретные или сливающиеся) \\
\hline Ретикулярные уплотнения интерстиция & В-линии (мультифокальные, дискретные) \\
\hline Субплевральная консолидация & Субплевральная консолидация \\
\hline Сегментарная (долевая) консолидация & Сегментарная (долевая) консолидация \\
\hline Воздушные бронхи на фоне признака консолидации & Признак воздушной бронхограммы \\
\hline Симптом «булыжной мостовой» & В-линии (мультифокальные, дискретные или сливающиеся), сочетающи- \\
& еся с субплевральной консолидацией \\
\hline Свободная жидкость в плевральной полости & Свободная жидкость в плевральной полости \\
\hline
\end{tabular}

Для определения диагностических возможностей УЗИ легких, оценки чувствительности и специфичности по сравнению с КТ органов грудной клетки проведена оценка частоты повреждения сегментов легких признаками инфильтрации и консолидации.

Признак уплотнения легочной паренхимы по типу «матового стекла» при КТ встретился у 100 \% исследуемых пациентов. Пневмония, вызванная COVID-19, носила полисегментарный характер с наиболее частым развитием в заднебазальных отделах легких и билатеральным повреждением. Частота выявления признака «матового стекла» при КТ и соответствующего ему признака В-линий при УЗИ представлена в табл. 3.

По количеству пораженных сегментов признаком инфильтрации раздельно в правом, левом легком, а также по общему количеству сегментов, определенных с помощью КТ и УЗИ, статистических различий выявлено не было (табл. 4).

Для оценки диагностической возможности УЗИ легких в верификации интерстициального поражения выполнен ROC-анализ по «золотому стандарту» КT с построением ROC-кривой и расчетом координатных точек.

У3-признак В-линий позволил с высокой точностью определять интерстициальные поражения с чувствительностью 87,9 \% и специфичностью 91,5 \% при выявлении инфильтрации в 3 сегментах и более (табл. 5). Площадь под ROC-кривой $-0,939, p<0,001$ (рис. 5).
Признак консолидации встречался реже и был не у всех пациентов. Частота выявления признака консолидации была статистически значимо меньше, чем признака инфильтрации ( $\mathrm{U}=35255,0 ; \mathrm{Z}=-12,950 ; p<0,001)$. Частота выявления признака консолидации при КТ легких и УЗ-признака консолидации представлена в табл. 6.

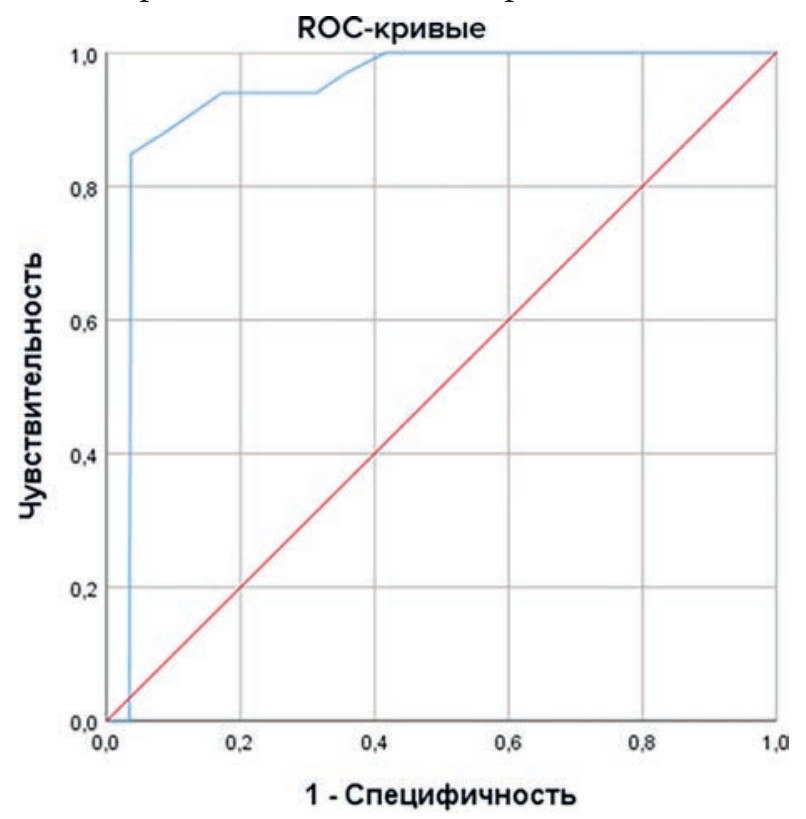

Pис. 5. ROC-кривая для признака инфильтрации легких Fig. 5. ROC curves for lung infiltration 
Таблица 3. Распределение встречаемости (количество пораженных сегментов) признака «матового стекла» при КТ и признака В-линий при УЗИ легких $(n=388)$

Table 3. Frequency of occurrence (number of affected segments) ground-glass pattern of CT and US B-lines $(n=388)$

\begin{tabular}{|c|c|c|c|c|c|}
\hline \multirow{2}{*}{ Легкое } & \multirow{2}{*}{ Сегмент } & \multicolumn{2}{|c|}{$\mathrm{KT}(n=388)$} & \multicolumn{2}{|c|}{ Узи $(n=388)$} \\
\hline & & aбc. & $\%$ & aбc. & $\%$ \\
\hline \multirow{10}{*}{$\begin{array}{l}\text { Правое } \\
\text { легкое }\end{array}$} & S1 & 158 & 40,70 & 162 & 41,8 \\
\hline & S2 & 185 & 47,7 & 194 & 50,0 \\
\hline & S3 & 204 & 52,6 & 212 & 54,6 \\
\hline & S4 & 183 & 47,2 & 186 & 47,9 \\
\hline & S5 & 193 & 49,7 & 198 & 51,0 \\
\hline & S6 & 238 & 61,3 & 242 & 62,4 \\
\hline & S7 & 156 & 40,2 & 153 & 39,4 \\
\hline & S8 & 210 & 54,1 & 221 & 57,0 \\
\hline & S9 & 265 & 68,3 & 273 & 70,4 \\
\hline & S10 & 279 & 71,9 & 280 & 72,2 \\
\hline \multirow{8}{*}{$\begin{array}{l}\text { Левое } \\
\text { легкое }\end{array}$} & $\mathrm{S} 1 / 2$ & 206 & 53,1 & 207 & 53,4 \\
\hline & S3 & 191 & 49,2 & 195 & 50,3 \\
\hline & S4 & 162 & 41,8 & 165 & 42,5 \\
\hline & S5 & 194 & 50,0 & 198 & 51,0 \\
\hline & S6 & 241 & 62,1 & 247 & 63,7 \\
\hline & $\mathrm{S} 7+8$ & 211 & 54,4 & 219 & 56,4 \\
\hline & S9 & 251 & 64,7 & 265 & 68,3 \\
\hline & $\mathrm{S} 10$ & 270 & 69,6 & 276 & 67,1 \\
\hline
\end{tabular}

При оценке количества пораженных сегментов признаком консолидации раздельно в правом, левом легком, общего количества сегментов, определенных с помощью КТ и УЗИ, статистических различий выявлено не было (табл. 7).

При выявлении признака консолидации в 5 легочных сегментах и более УЗИ легких демонстрирует высокую чувствительность и специфичность - 84,6 и 79,2 \% соответственно (табл. 8). Площадь под ROC-кривой $0,846, p<0,001$ (рис. 6).

\section{Обсуждение}

Прикроватные фокусированные УЗИ используют в различных клинических ситуациях, связанных с возникновение дыхательной, сердечно-сосудистой недо-
Таблица 4. Количество пораженных сегментов признаком инфильтрации $(n=388)$

Table 4. Number of affected segments with lung infiltration $(n=388)$

\begin{tabular}{|c|c|c|c|}
\hline & $\begin{array}{c}\text { KT } \\
\mathrm{Me}(\mathrm{Q} 1-\mathrm{Q} 3)\end{array}$ & $\begin{array}{c}\text { Узи } \\
\text { Me (Q1-Q3) }\end{array}$ & $\begin{array}{c}\text { Критерий } \\
\text { Манна-Уитни }\end{array}$ \\
\hline $\begin{array}{l}\text { Правое } \\
\text { легкое }\end{array}$ & $5,0(2,0-9,0)$ & $5,0(2,0-9,5)$ & $\begin{array}{l}U=73761,50 \\
Z=-0,49 \\
P=0,63\end{array}$ \\
\hline $\begin{array}{l}\text { Левое } \\
\text { легкое }\end{array}$ & $4,0(2,0-8,0)$ & $4,0(2,0-8,5)$ & $\begin{array}{l}U=73357,00 \\
Z=-0,62 \\
P=0,53\end{array}$ \\
\hline Сумма & $9,0(4,0-16,0)$ & $9,0(5,0-15,0)$ & $\begin{array}{l}U=73324,50 \\
Z=-0,63 \\
P=0,53\end{array}$ \\
\hline
\end{tabular}

Таблица 5. Координаты ROC-кривой для признака инфильтрации легких

Table 5. Coordinates of ROC curves for lung infiltration

\begin{tabular}{|lll|}
\hline $\begin{array}{c}\text { Верно, если боль- } \\
\text { ше или равно }\end{array}$ & Чувствительность & 1-Специфичность \\
\hline 1,000 & 0,000 & 0,034 \\
\hline 2,000 & 0,848 & 0,037 \\
\hline 3,000 & 0,879 & 0,085 \\
\hline 4,000 & 0,939 & 0,172 \\
\hline 5,000 & 0,939 & 0,245 \\
\hline 6,000 & 0,939 & 0,313 \\
\hline 7,000 & 0,970 & 0,358 \\
\hline 8,000 & 1,000 & 0,417 \\
\hline 9,000 & 1,000 & 0,468 \\
\hline 10,000 & 1,000 & 0,515 \\
\hline 11,000 & 1,000 & 0,561 \\
\hline 12,000 & 1,000 & 0,589 \\
\hline 13,000 & 1,000 & 0,628 \\
\hline 14,000 & 1,000 & 0,642 \\
\hline 15,000 & 1,000 & 0,679 \\
\hline 16,000 & 1,000 & 0,744 \\
\hline 17,000 & 1,000 & 0,783 \\
\hline 18,000 & 1,000 & 1,000 \\
\hline
\end{tabular}


Таблица 6. Распределение встречаемости признака консолидации (количество пораженных сегментов), $n=388$

Table 6. Frequency of consolidation (number of affected segments), $n=388$

\begin{tabular}{|c|c|c|c|c|c|}
\hline \multirow{2}{*}{ Легкое } & \multirow{2}{*}{ Сегмент } & \multicolumn{2}{|c|}{ KT } & \multicolumn{2}{|c|}{ Узи } \\
\hline & & a6c. & $\%$ & $\mathrm{a} \sigma c$. & $\%$ \\
\hline \multirow{10}{*}{$\begin{array}{l}\text { Пра- } \\
\text { вое } \\
\text { легкое }\end{array}$} & S1 & 74 & 19,10 & 70 & 18,0 \\
\hline & S2 & 88 & 22,7 & 87 & 22,4 \\
\hline & S3 & 84 & 21,6 & 83 & 21,4 \\
\hline & S4 & 80 & 20,6 & 79 & 20,4 \\
\hline & S5 & 79 & 20,4 & 80 & 20,6 \\
\hline & S6 & 110 & 28,4 & 110 & 28,4 \\
\hline & S7 & 74 & 19,1 & 62 & 16,0 \\
\hline & S8 & 99 & 25,5 & 102 & 26,3 \\
\hline & S9 & 121 & 31,2 & 132 & 34,0 \\
\hline & S10 & 127 & 32,7 & 131 & 33,8 \\
\hline \multirow{8}{*}{$\begin{array}{l}\text { Левое } \\
\text { легкое }\end{array}$} & $\mathrm{S} 1 / 2$ & 102 & 26,3 & 100 & 25,8 \\
\hline & S3 & 82 & 21,1 & 84 & 21,6 \\
\hline & S4 & 71 & 18,3 & 72 & 18,6 \\
\hline & S5 & 95 & 24,5 & 94 & 24,2 \\
\hline & S6 & 114 & 29,4 & 114 & 29,4 \\
\hline & $S 7+8$ & 101 & 26,0 & 104 & 26,8 \\
\hline & S9 & 121 & 31,2 & 132 & 34,0 \\
\hline & S10 & 128 & 33,0 & 133 & 34,3 \\
\hline
\end{tabular}

Таблица 7. Количество пораженных сегментов признаком консолидации $(n=388)$

Table 7. Number of affected segments with lung consolidation $(n=388)$

\begin{tabular}{|c|c|c|c|}
\hline & $\begin{array}{c}\text { KT } \\
\mathrm{Me}(\mathrm{Q} 1-\mathrm{Q} 3)\end{array}$ & $\begin{array}{c}\text { Узи } \\
\text { Me (Q1-Q3) }\end{array}$ & $\begin{array}{c}\text { Критерий } \\
\text { Манна-Уитни }\end{array}$ \\
\hline $\begin{array}{l}\text { Правое } \\
\text { легкое }\end{array}$ & $0,0(0,0-4,0)$ & $0,0(0,0-4,0)$ & $\begin{array}{l}U=74108,50 \\
Z=-0,41 \\
P=0,69\end{array}$ \\
\hline $\begin{array}{l}\text { Левое } \\
\text { легкое }\end{array}$ & $1,0(0,0-4,0)$ & $1,0(1,0-4,0)$ & $\begin{array}{l}U=73732,00 \\
Z=-0,54 \\
P=0,59\end{array}$ \\
\hline Сумма & $1,0(0,0-8,0)$ & $1,0(1,0-8,0)$ & $\begin{array}{l}U=74024,50 \\
Z=-0,42 \\
P=0,67\end{array}$ \\
\hline
\end{tabular}

Таблица 8. Координаты ROC-кривой для признака консолидации легких

Table 8. Coordinates of ROC curves for lung consolidation

\begin{tabular}{|ccc|}
\hline $\begin{array}{c}\text { Верно, если боль- } \\
\text { ше или равно }\end{array}$ & Чувствительность & 1-Специфичность \\
\hline$-1,000$ & 0,000 & 0,000 \\
\hline 0,500 & 0,154 & 0,109 \\
\hline 1,000 & 0,538 & 0,123 \\
\hline 2,000 & 0,615 & 0,141 \\
\hline 3,000 & 0,615 & 0,157 \\
\hline 4,000 & 0,615 & 0,163 \\
\hline 5,000 & 0,846 & 0,208 \\
\hline 6,000 & 0,923 & 0,251 \\
\hline 7,000 & 0,923 & 0,301 \\
\hline 8,000 & 0,923 & 0,365 \\
\hline 9,000 & 1,000 & 0,429 \\
\hline 11,000 & 1,000 & 1,000 \\
\hline
\end{tabular}

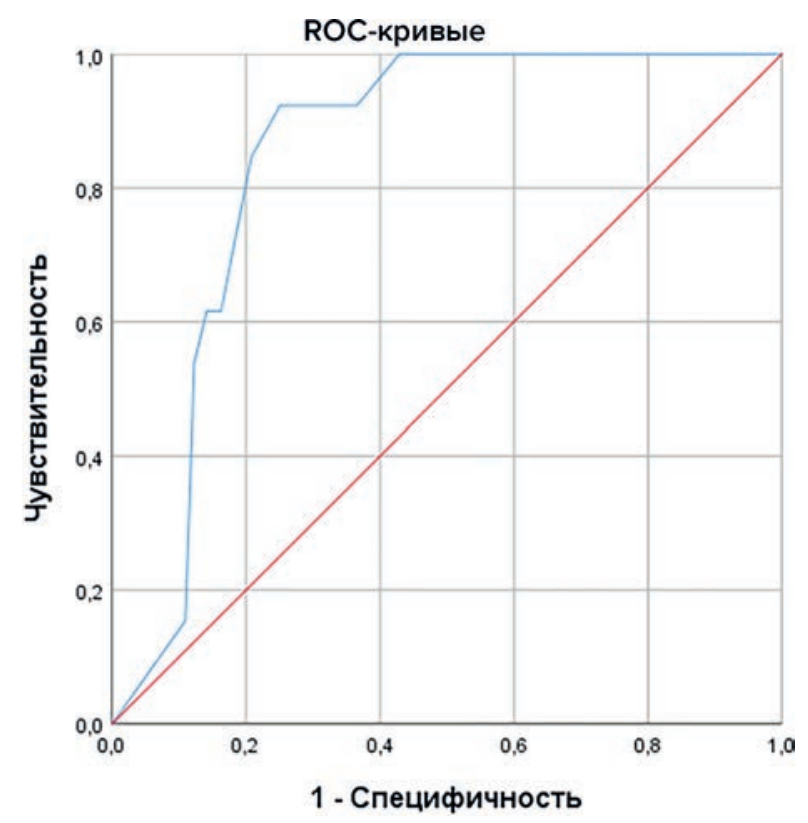

Pиc. 6. ROC-кривая для признака консолидации легких

Fig. 6. ROC curves for lung consolidation

статочности и других осложнений COVID-19 [19, 20]. В этом исследовании УЗИ легких продемонстрировало высокую диагностическую точность, сравнимую с КТ легких, как для выявления легочных паттернов, так и для описания распределения заболевания в различных областях легких у пациентов с пневмонией, вызванной COVID-19. 
Одной из первых работ, показывающих корреляцию между КТ и УЗИ, было исследование Nouvenne A. et al. (2020), которое продемонстрировало значимость их использования на ранних стадиях заболевания [10]. Последовавшие за ним сравнительные исследования в различных странах также отметили высокую степень сопоставимости У3-данных и результатов КТ [15, 21, 22]. Soldati et al. (2020) в своем исследовании предложили использовать УЗИ для сортировки пациентов с симптомами COVID-19 и пневмонией на догоспитальном этапе, что значительно упростит работу в условиях пандемии [23, 24].

У пациентов с альвеолярно-интерстициальной пневмонией, вызванной COVID-19, выявляемые УЗ-признаки были ассоциированы с изменениями КТ органов грудной клетки. В нашем исследовании наиболее частыми УЗ-признаками были очаговые участки интерстициального синдрома (отдельные и сливные В-линии) с возможным наличием мелких, множественных субплевральных уплотнений и изменений плевральной линии, которые отражали изменения на КТ в виде уплотнения легочной паренхимы по типу «матового стекла», утолщенной плевры. На втором месте по частоте развития стоял УЗ-признак консолидации с воздушными бронхограммами, которому на КТ соответствовал также признак консолидации легочной ткани, в то время как плевральный выпот присутствовал лишь в нескольких случаях. В исследовании Nouvenne A. et al. (2020) наиболее типичными У3-признаками были различные по площади зоны интерстициального синдрома (сливные или дискретные В-линии) с возможным наличием мелких или крупных субплевральных консолидаций и изменений плевральной линии; плевральный выпот в этом исследовании также обнаруживали редко[10]. В исследовании Ökmen K. et al. (2020) признаки повреждения легких при COVID-19 присутствовали у 72,5 \% пациентов при КТ и у 78,4 \% пациентов при УЗИ. Так же как и в нашем исследовании, наиболее часто выявляемыми признаками были инфильтрация и консолидация.

Посегментного анализа с помощью ультразвука в литературе нам обнаружить не удалось. Как правило, для УЗИ легких характерны исследования в зонах, долях легкого. Что касается частоты встречаемости пораженных зон легких, то при COVID-19 чаще всего, так же как и в нашем исследовании, это заднебазальные отделы и билатеральное поражение. Исследование Chung M. et al. (2019) показало, что в правом легком верхняя доля поражается в $67 \%$, средняя доля $-57 \%$, нижняя доля $-76 \%$. В левом легком верхняя доля вовлечена в патологический процесс в 67 \% случаев, нижняя доля - 67 \%. Билатеральное поражение легочной паренхимы было выявлено в 76 \% случаев, а одностороннее (преимущественно справа) наблюдалось у $10 \%$ [25].

Полученные в нашем исследовании результаты согласуются с исследованиями, экспертными заключениями и сериями случаев, ранее опубликованными в литературе $[3,15,17,26]$. Значимая связь между показателями УЗИ и КТ позволяет сделать шаг вперед в определении роли ультразвука легких в диагностике и оценке пневмонии, вызванной COVID-19.

\section{Заключение}

Использование УЗИ легких во время пандемии COVID-19 позволяет выявить и оценить объем и характер повреждения легочной ткани с высокой степенью чувствительности и специфичности. УЗИ легких является хорошим прикроватным вспомогательным методом, но лишь при его должном применении подготовленным персоналом, особенно в отношении соблюдения методики исследования и уверенной проекции сегментов легких на грудную стенку. По мере накопления опыта как в других, так и в нашем исследовании верифицированы основные УЗ-признаки патологических изменений, такие как В-линии и консолидация, которые были ассоциированы с аналогичными изменениями на КТ. Чувствительность УЗИ легких в точности посегментной диагностики поражения признаком инфильтрации составляет 87,9 \%, а специфичность 91,5 \%. Чувствительность УЗ-признака консолидации составила 84,6 \%, специфичность 79,2 \% соответственно. Таким образом, УЗИ легких продемонстрировало точность, сравнимую с КТ органов грудной клетки, при выявлении повреждения легких у пациентов с COVID-19.

Конфликт интересов. Авторы заявляют об отсутствии конфликта интересов.

Вклад авторов. Лахин Р.Е., Жирнова Е.А., Щеголев А.В., Йованикич О., Железняк И.С., Меньков И.А., Салухов В.В., Чугунов. А.А. - разработка концепции статьи, получение и анализ фактических данных, написание и редактирование текста статьи, проверка и утверждение текста статьи.

\section{ORCID авторов}

Лахин Р.Е. - 0000-0001-6819-9691

Жирнова Е.А. - 0000-0003-1865-3838

Щеголев А.В. - 0000-0001-6431-439X

Йованикич О. - 0000-0002-6795-3143

Железняк И.С. - 0000-0001-7383-512Х

Меньков И.А. - 0000-0002-1569-2180

Салухов В.В. - 0000-0003-1851-0941

Чугунов А.А. - 0000-0002-2532-6133 


\section{Литература/References}

[1] Hu Z., Liu Z., Dong Y., et al. Evaluation of lung involvement in COVID-19 pneumonia based on ultrasound images. Biomed Eng Online. 2021; 20: 27. DOI: 10.1186/s12938-021-00863-x.

[2] Peris A., Zagli G., Barbani F., et al. The value of lung ultrasound monitoring in $\mathrm{H} 1 \mathrm{~N} 1$ acute respiratory distress syndrome. Anaesthesia. 2010; 65: 294-7. DOI: 10.1111/j.1365-2044.2009.06210.x.

[3] Convissar D.L., Gibson L.E., Berra L., et al. Application of Lung Ultrasound during the COVID-19 Pandemic: A Narrative Review. Anesth Analg. 2020: 345-50. DOI: 10.1213/ANE.0000000000004929.

[4] Bertsimas D., Lukin G., Mingardi L., et al. COVID-19 mortality risk assessment: An international multi-center study. PLoS One. 2020; 15. DOI: $10.1371 /$ journal.pone.0243262.

[5] Dargent A., Chatelain E., Kreitmann L., et al. Lung ultrasound score to monitor COVID-19 pneumonia progression in patients with ARDS. PLoS One. 2020; 15. DOI: 10.1371/journal.pone.0236312.

[6] Ramos Hernández C., Botana Rial M., et al. Lung Ultrasound to Predict Unfavorable Progress in Patients Hospitalized for COVID-19. Arch Bronconeumol. 2021; 57: 47-54. DOI: 10.1016/j.arbres.2020.07.043.

[7] Buonsenso D., Raffaelli F., Tamburrini E., et al. Clinical role of lung ultrasound for diagnosis and monitoring of COVID-19 pneumonia in pregnant women. Ultrasound Obstet Gynecol. 2020; 56: 106-9. DOI: 10.1002/uog.22055.

[8] Giannini A., Mantovani A., Vezzoli C., et al. Lung ultrasound for pregnant women admitted to ICU for COVID-19 pneumonia. Minerva Anestesiol. 2020; 86: 1248-9. DOI: 10.23736/S0375-9393.20.14726-6.

[9] Kumar A., Weng Y., Duanmu Y., et al. Lung Ultrasound Findings in Patients Hospitalized With COVID-19. J Ultrasound Med. 2021: jum.15683. DOI: 10.1002/jum.15683.

[10] Nouvenne A., Zani M.D., Milanese G., et al. Lung Ultrasound in COVID-19 Pneumonia: Correlations with Chest CT on Hospital admission. Respiration. 2020; 99: 617-24. DOI: 10.1159/000509223.

[11] Volpicelli G., Gargani L., Perlini S., et al. Lung ultrasound for the early diagnosis of COVID-19 pneumonia: an international multicenter study on behalf of the International Multicenter Study Group on LUS in COVID-19. Intensive Care Med. 2021. DOI: 10.1007/s00134-021-06373-7.

[12] Manivel V., Lesnewski A., Shamim S., et al. CLUE: COVID-19 lung ultrasound in emergency department. EMA - Emerg Med Australas. 2020; 32: 694-6. DOI: 10.1111/1742-6723.13546.

[13] Smith M.J., Hayward S.A., Innes S.M., Miller A.S.C. Point-of-care lung ultrasound in patients with COVID-19 - a narrative review. Anaesthesia. 2020; 75: 1096-104. DOI: 10.1111/anae.15082.

[14] Rizzetto F., Perillo N., Artioli D., et al. Correlation between lung ultrasound and chest CT patterns with estimation of pulmonary burden in COVID-19 patients. Eur J Radiol. 2021; 138: 109650. DOI: 10.1016/j.ejrad.2021.109650.

[15] Ökmen K., Yıldız D.K., Soyaslan E. Comparison of lung ultrasonography findings with chest computed tomography results in coronavirus (COVID-19) pneumonia. J Med Ultrason. 2021. DOI: 10.1007/s10396-021-01081-7.

[16] McDermott C., Łącki M., Sainsbury B., et al. Sonographic Diagnosis of COVID-19: A Review of Image Processing for Lung Ultrasound. Front Big Data. 2021; 4: 612561. DOI: 10.3389/fdata.2021.612561.

[17] Soldati G., Smargiassi A., Inchingolo R., et al. Proposal for International Standardization of the Use of Lung Ultrasound for Patients With COVID-19. J Ultrasound Med. 2020; 39: 1413-9. DOI: 10.1002/jum.15285.

[18] Лахин Р.Е., Жирнова Е.А. Способ фокусированного ультразвукового исследования легких (Русский протокол). В кн.: Усовершенствование способов и аппаратуры, применяемых в учебном процессе, медико-биологических исследованиях и клинической практике. Под общей ред. проф. Котива Б.Н. СПб: ВМедА, 2021. C. 58-9. [Lakhin R.E., Zhirnova E.A. Sposob fokusirovannogo ul'trazvukovogo issledovaniya legkih (Russkij protokol). In: Usovershenstvovanie sposobov i apparatury, primenyaemyh $v$ uchebnom processe, mediko-biologicheskih issledovaniyah i klinicheskoj praktike. Pod obshchej red. prof. Kotiva B.N. SPb: VMedA, 2021. P. 58-9. (In Russ)]

[19] Hussain A., Via G., Melniker L., Goffi A., et al. Multi-organ point-of-care ultrasound for COVID-19 (PoCUS4COVID): international expert consensus. Crit Care. 2020; 24(1): 702. DOI: 10.1186/s13054-020-03369-5.

[20] Alharthy A., Faqihi F., Abuhamdah M., et al. Prospective Longitudinal Evaluation of Point-of-Care Lung Ultrasound in Critically III Patients With Severe COVID-19 Pneumonia. J Ultrasound Med. 2021; 40: 443-56. DOI: 10.1002/jum.15417.

[21] Tung-Chen Y., Martí de Gracia M., et al. Correlation between Chest Computed Tomography and Lung Ultrasonography in Patients with Coronavirus Disease 2019 (COVID-19). Ultrasound Med Biol. 2020; 46: 2918-26. DOI: 10.1016/j.ultrasmedbio.2020.07.003.

[22] Karacaer C., Karabay O., Gunduz Y., et al. Correlation of lung ultrasound and computed tomography findings in COVID-19 pneumonia. J Coll Physicians Surg Pakistan. 2021; 30: S147-52. DOI: 10.29271/jcpsp.2020.Supp2.S147.

[23] Soldati G., Smargiassi A., Inchingolo R., et al. On Lung Ultrasound Patterns Specificity in the Management of COVID-19 Patients. J Ultrasound Med. 2020; 39: 2283-4. DOI: 10.1002/jum.15326.

[24] Soldati G., Smargiassi A., Inchingolo R., et al. Is There a Role for Lung Ultrasound During the COVID-19 Pandemic? J Ultrasound Med. 2020; 39: 1459-62. DOI: 10.1002/jum.15284.

[25] Chung M., Bernheim A., Mei X., et al. CT imaging features of 2019 novel coronavirus (2019-NCoV). Radiology. 2020; 295: 202-7. DOI: 10.1148/radiol.2020200230.

[26] Gargani L., Soliman-Aboumarie H., et al. Why, when, and how to use lung ultrasound during the COVID-19 pandemic: Enthusiasm and caution. Eur Heart J Cardiovasc Imaging. 2020; 21: 941-8. DOI: 10.1093/ehjci/jeaa163. 